\title{
A comparison of the JO-IMRT dose distribution calculated by the SOURCE 8 and 20 in the DOSXYZnre for head-and-neck cancer
}

\author{
D.T. Tai ${ }^{*}$, H.D. Tuan ${ }^{2}$, L.T. Oanh ${ }^{3}$, H. Naeem ${ }^{4}$, T.T.H. Loan ${ }^{5,6}$, S. Yani ${ }^{7}$ \\ ${ }^{1}$ Department of Industrial Electronics and Biomedical Engineering, Faculty of Electrical and Electronic Engineering, \\ Ho Chi Minh City University of Technology and Education, Ho Chi Minh, 749000, Vietnam \\ ${ }^{2}$ Department of Oncology, University Medical Shing Mark Hospital, Bien Hoa, 810000, Vietnam \\ ${ }^{3}$ Department of Radiation Technology, Ho Chi Minh City Oncology Hospital, Ho Chi Minh, 702000, Vietnam \\ ${ }^{4}$ Department of Physics, Division of Science and Technology, University of Education, Lahore, 54000, Pakistan \\ ${ }_{5}^{5}$ Faculty of Physics \& Engineering Physics, VNUHCM-University of Science, 749000, Vietnam \\ ${ }^{6}$ Vietnam National University Ho Chi Minh City, 749000, Vietnam \\ ${ }^{7}$ Department of Physics, Faculty of Mathematic and Natural Sciences, IPB University, Bogor 16680, Indonesia
}

\section{- Original article}

*Corresponding author:

Duong Thanh Tai, Ph.D.,

E-mail: taidt@hcmute.edu.vn

Revised: December 2020

Accepted: January 2021

Int. J. Radiat. Res., October 2021; 19(4): $853-860$

DOI: $10.29242 /$ ijrr.19.4.853

\section{ABSTRACT}

Background: The purpose of this study is to compare SOURCE 8 and 20 in the EGSnrc-based DOSXYZnrc Monte Carlo code for Jaws-only intensitymodulated radiation therapy (JO-IMRT) dose distribution, and demonstrate the advantage of SOURCE 20 to SOURCE 8 to treat head-and-neck cancer. Materials and Methods: The clinical photon beams of the HPD Siemens Primus linear accelerator simulated using the BEAMnrc code and then verified by measurement. The phase-space files generated by the BEAMnrc code were used as an input for the DOSXYZnrc to calculate the JO-IMRT dose distributions of patients (in form of CT images) using the SOURCES 8 and 20. The isodose distribution on slices, DVH and gamma index $(3 \% / 3 \mathrm{~mm}, 2 \% / 2$ $\mathrm{mm}$, and $1 \% / 1 \mathrm{~mm}$ ) were compared with Monte Carlo and treatment planning system (TPS) results. Furthermore, the efficient computation of dose distributions such as time running, working load and uncertainty error calculation also considered for evaluation. Results: JO-IMRT dose distributions calculated by SOURCE 8 and 20 compared with the Prowess Panther TPS. The time running, working load based on SOURCE 20 were significantly less than SOURCE 8. Futhermore, the uncertainty calculation based on SOURCE 20 was also significantly less than SOURCE 8. Conclusion: The JO-IMRT dose distributions calculated by SOURCE 20 are more efficient than SOURCE 8.

Keyword: JO - IMRT plan, Monte Carlo simulation, SOURCE 8, SOURCE 20, EGSnrC.

\section{INTRODUCTION}

The main objective of radiotherapy is to kill tumor cells to the utmost and save the normal surrounding healthy tissues. These treatments need exact finding of all those dosimetric parameters, which are compulsory for treatment planning system (TPS). Clinically, many TPS calculate radiation dose using analytical method, which is not accurate in inhomogeneous medium (1-3). Alternatively, the Monte Carlo (MC) simulation known to be the most accurate method for radiotherapy treatment planning and dose calculation. MC is one of the most powerful methods (sometimes referred to as major approach) for the calculation of dose distribution and also used as a benchmark to validate the dose distribution performed by any TPS $(4,5)$.

MC simulation is a statistical method that uses 
random numbers to simulate and contain data of every particle in beam including the charge, total energy, position, spatial and angular distribution etc., and has the ability to accurately model the complex geometries to predict the dose distribution in patient's heterogeneous anatomy $(6,7)$. Many general-purpose $\mathrm{MC}$ codes are available for radiotherapy and its applications such as IMRT, VMAT, and so on (8-10). Benhalouche et al. (2013) have successfully used GATE/Geant4 MC code to evaluate dose distribution of patient IMRT treatment planning. Their results show that the GATE code can be used to simulate the IMRT planning with high accuracy (11).

The EGSnrc-based code successfully used to verify the SABR VMAT treatment planning by Bergman et al. (2014). The 3D gamma factors showed that more than $99 \%$ voxels pass for the $3 \% / 3 \mathrm{~mm}$ criteria (12). The most validated and used code is the EGSnrc (13). It has been widely used for simulation of radiotherapy beams in medical physics for many different treatment techniques. The EGSnrc has two main sub-codes, namely, the BEAMnrc and DOSXYZnrc, which can be used for designing and simulation of complex accelerator treatment head geometries, and calculating the dose distribution, respectively $(8,14,15)$.

To calculate dose distribution using the DOSXYZnrc, 12 different SOURCE types are used and each SOURCE has different features (e.g. point SOURCE, phase-space SOURCE incident from any direction etc.) for independent simulation. Among these 12 SOURCE, SOURCE 20 developed by Lobo and Popescu has been validated for IMRT dose distributions $(13,16,17)$. Comparison between SOURCE 20 and 8 for head -and-neck treatment plans suggest that simulation can be performed in a single run with SOURCE 20 instead of seven runs with SOURCE 2 (16).

Similar comparison between SOURCE 20 and 21 suggests using SOURCE 20 for IMRT dose calculations (13, 17). Yani et al. (16) reported utilization of SOURCE 20 to simulate IMRT planning with dynamic MLC. They found that the DVH for total body of patient in the simulation has a same pattern with the DVH from AAA.
Futhermore, Linares et al compared between SOURCE 20 and 21. Their result shows that SOURCE 20 is better than SOURCE 21 (17). While the scientific paper related to comparison of SOURCE 20 and 21 published in breast irradiation, there is no recent publication in comparision of SOURCE 8 and 20 for calculating for JO-IMRT technique.

The aim of this study was to: (i) compare SOURCE 8 and 20 in the EGSnrc-based DOSXYZnrc MC code for JO-IMRT dose distribution to authenticate the advantage of using one SOURCE over another, and (ii) to improve the calculation efficiency and minimize errors while ensuring accuracy in simulation for head-and-neck cancer. The evaluation has been done by comparing the dosimetric parameters such as gamma index and dose volume histogram (DVH) for both SOURCEs. Furthermore, the simulation performed on the same computer in order to choose the most suitable SOURCE to achieve high efficiency and to reduce the simulation time.

\section{MATERIALS AND METHODS}

\section{JO-IMRT plan on prowess panther TPS}

The MC has been used to calculate dose distribution of head-and-neck IMRT treatment planning using the Collapsed Cone Convolution (CCC) method on the Prowess Panther TPS at Dong Nai General Hospital. Details information about the plan is as follows:

- Photon beam energy: $6 \mathrm{MV}$

- Collimator: $\theta_{\mathrm{C}}=0^{0}$.

- The patient table does not move, only the accelerator locates around the patient table: $\theta_{\mathrm{T}}=0^{0}$

- There are 7 treatment beams with gantry angles $\left(\theta_{\mathrm{G}}\right): 72^{0} ; 100^{0} ; 155^{0} ; 210^{0} ; 252^{0} ; 265^{0}$, and $300^{\circ}$.

- Source-to-surface distance (SSD) of the 7 beams: $91.6 \mathrm{~cm} ; 91.4 \mathrm{~cm} ; 89.1 \mathrm{~cm}$; $90.3 \mathrm{~cm}$; $92.4 \mathrm{~cm} ; 92.7 \mathrm{~cm}$, and $92.9 \mathrm{~cm}$.

Each beam consists of 7 segments with the different beam segments. A total of 49 segments were needed to simulate (18).

Int. J. Radiat. Res., Vol. 19 No. 4, October 2021 


\section{Monte Carlo simulation}

The EGSnrc-based BEAMnrc code was used to; (i) model linear accelerator treatment head, (ii) simulate photon beam and create a phase-space file (a phase-space file contains particle information including position, energy, etc). The DOSXYZnrc code is used to calculate the radiation dose as per the CT imaging data of patients (19).

\section{Simulating a HPD siemens primus}

The 6 MV photon beam of the HPD Siemens Primus linear accelerator was modeled using the EGSnrc-based BEAMnrc MC code. The primus accelerator consists of 7 main component modules (CMs), namely, vacuum envelope (SLABS), the target (SLABS), the flattening filter (FLATFILT), chambers for monitoring the linac output (CHAMBER), the mirror (MIRROR), the jaws $\mathrm{X}$ and jaws $\mathrm{Y}$ (JAWS). The structure and the CMs of the accelerator shown in figure 1.

After the MC simulation, the realistic photon beams were stored in phase-space files which have been used as input to the DOSXYZnrc to simulate dose distributions in the geometry of patients built by the CT data. The process of the simulation of the accelerator detailed in our previous studies $(9,18,20,21)$.

A total of $1 \times 10^{8}$ number of histories were simulated. Energy threshold (ESAVE) set to 2 $\mathrm{MeV}$ in all component modules and the variance reduction technique applied in our simulation is directional bremsstrahlung splitting (DBS) (8). MC transport parameters recommended by Walters and Rogers was used with electron and photon cut-off energies of $700 \mathrm{keV}$ and $10 \mathrm{keV}$, respectively $(14,15)$.

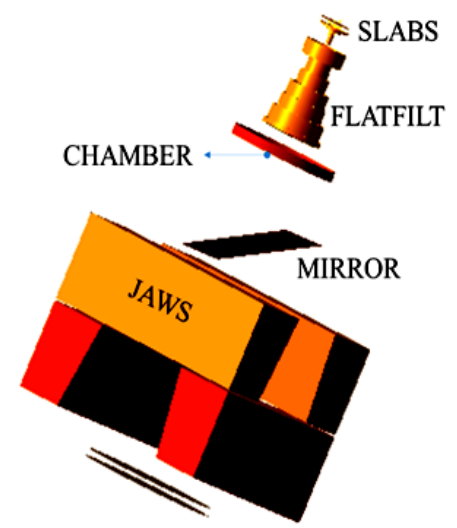

Figure 1. Simulation of the treatment head and CMs used in the BEAMnrc $6 \mathrm{MV}$ photon beam. The main structural components of the accelerator are, from top: initial electron from vacuum envelope, target, fattening filter, monitor ion chamber, mirror and jaws.

\section{SOURCE 8 and 20 configuration in the DOSXYZnrc}

DOSXYZnrc's SOURCE library includes 12 different SOURCES that can be used in the simulation such as parallel rectangular beam incident from any directions, phase-space SOURCE particles incident from any directions, point source incident from the front, phase-space source incident from multiple angles, etc. The characteristics and information of the sources described below, as the purpose of this work is to use SOURCE 8 and 20 only.

SOURCE 8 in the DOSXYZnrc is the phase-space source incident from multiple directions. This source uses a phase-space file generated from the BEAMnrc simulation at any flat scoring plane of a linear accelerator geometry. The user can choose any particular type of particles from the phase-space file and score dose components using the LATCH filter. The field size of the beam can be reduced using the parameter BEAM SIZE. Dose distributions are stored in the 3ddose file for every beam angle. The calculation of accumulative dose distribution from each beam must be calculated in order to obtain the real dose distribution.

SOURCE 20 developed by Lobo and Popescu (22) is synchronized phase-space source that allows users to simulate the continuous motion of the phase-space source relative to the DOSXYZnrc phantom over the specified ranges of incident directions, SSD and isocenter coordinates (23). It also allows users to enhance the capabilities of simulation with the phase-space SOURCE incident from multiple directions. Therefore, the dose distribution can be calculated for all beam angles at one time.

The simulation declaration in the case of using SOURCE 20 could be executed simultaneously for all segments of the beam, thereby significantly reducing the time for the simulation, the workload to perform as well as simplifying the process of declaration.

\section{Flowchart of the main process of Calculating of JO-IMRT dose distribution}

The simulation process is divided into two main steps (as shown in figure 2) to evaluate the difference between dose distributions by 
changing the source structure in the DOSXYZnrc library.

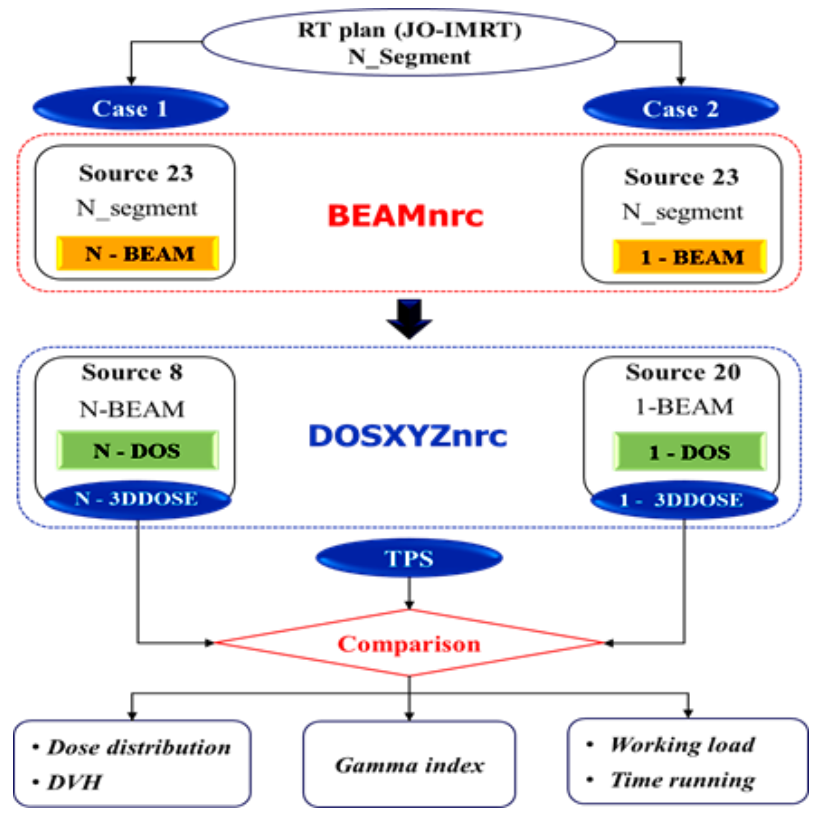

Figure 2. Flowchart showing the process of simulation.

Firstly, the radiation input to the BEAMnrc code is SOURCE 23 in the library (accelerator component containing the JAWX and JAWY CMs). The process of simulating dose distributions on phantom is done by using the DOSCTP. This software was built with the MATLAB program and the information about the projection fields of the treatment plan was defined here. The DOSCTP was linked to the EGSnrc-based DOSXYZnrc program and SOURCE 8 in the library of the DOSXYZnrc was used to calculate the dose distribution. Secondly, the same procedure was adopted (as discussed in the first step) for the BEAMnrc and SOURCE 20 instead of SOURCE 8 was used to declare the information of the beams used during the simulation and calculate the dose distribution on the patient.

Finally, to choose the most suitable source to achieve high efficiency and to reduce the simulation time, it is necessary to test and analyze these sources. For this purpose, both cases were simulated on the same computer, the declared information about treatment planning, input parameters and the interactive data information section of the material used is exactly the same for the two cases. The simulation was run on Windows 7 operation system with Intel Core i5-2400 processors, 4 GB Memory, and 1 TB Hard Drive.
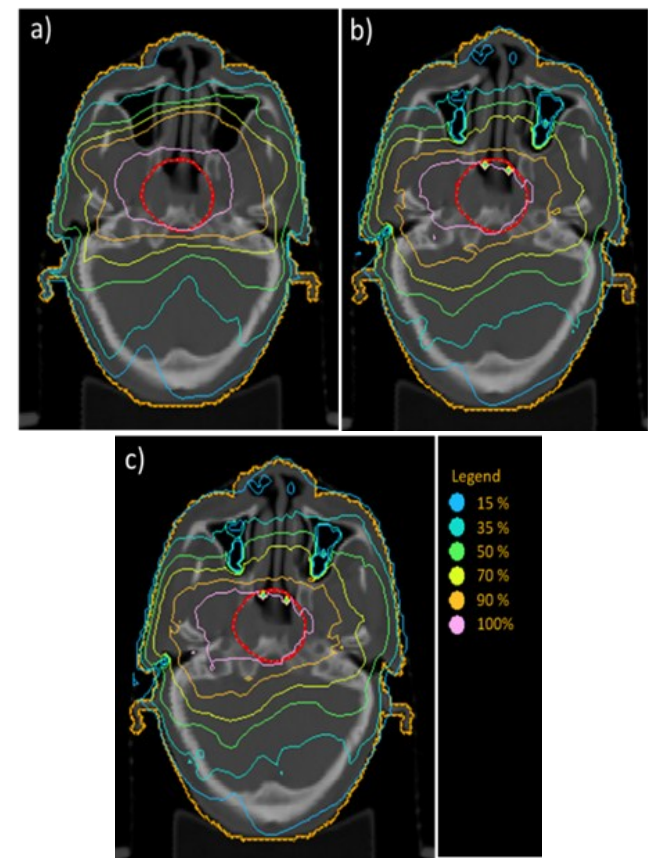

Figure 3. Comparison of the dose distribution on CT slices a) TPS, b) SOURCE 20, and SOURCE 8 of MC simulation.

\section{RESULTS}

\section{Dose distribution}

The $100 \%, 90 \%, 70 \%$ of isodose lines for TPS, SOURCE 20, and SOURCE 8 of MC simulation has a similar shape. However, the $15 \%, 35 \%, 50 \%$ of isodose lines for TPS are difference with SOURCE 20 and SOURCE 8 of MC simulation. This is because of dose perturbation by air cavities in megavoltage photon beams (24).

\section{Gamma index}

For 2D dose distribution, the Gamma index (for the same CT slice) between TPS, SOURCE 8, and SOURCE 20 (as shown in figures 4 and 5) with $3 \% / 3 \mathrm{~mm}$ criteria is $94.4 \%$ and $94.3 \%$ with $5.6 \%$ and $5.7 \%$ difference, respectively. While for 3D dose distribution, the Gamma index $(3 \% / 3 \mathrm{~mm})$ is $93.8 \%$ and $97.4 \%$ with $6.2 \%$ and $2.6 \%$ difference, respectively.

Int. J. Radiat. Res., Vol. 19 No. 4, October 2021 


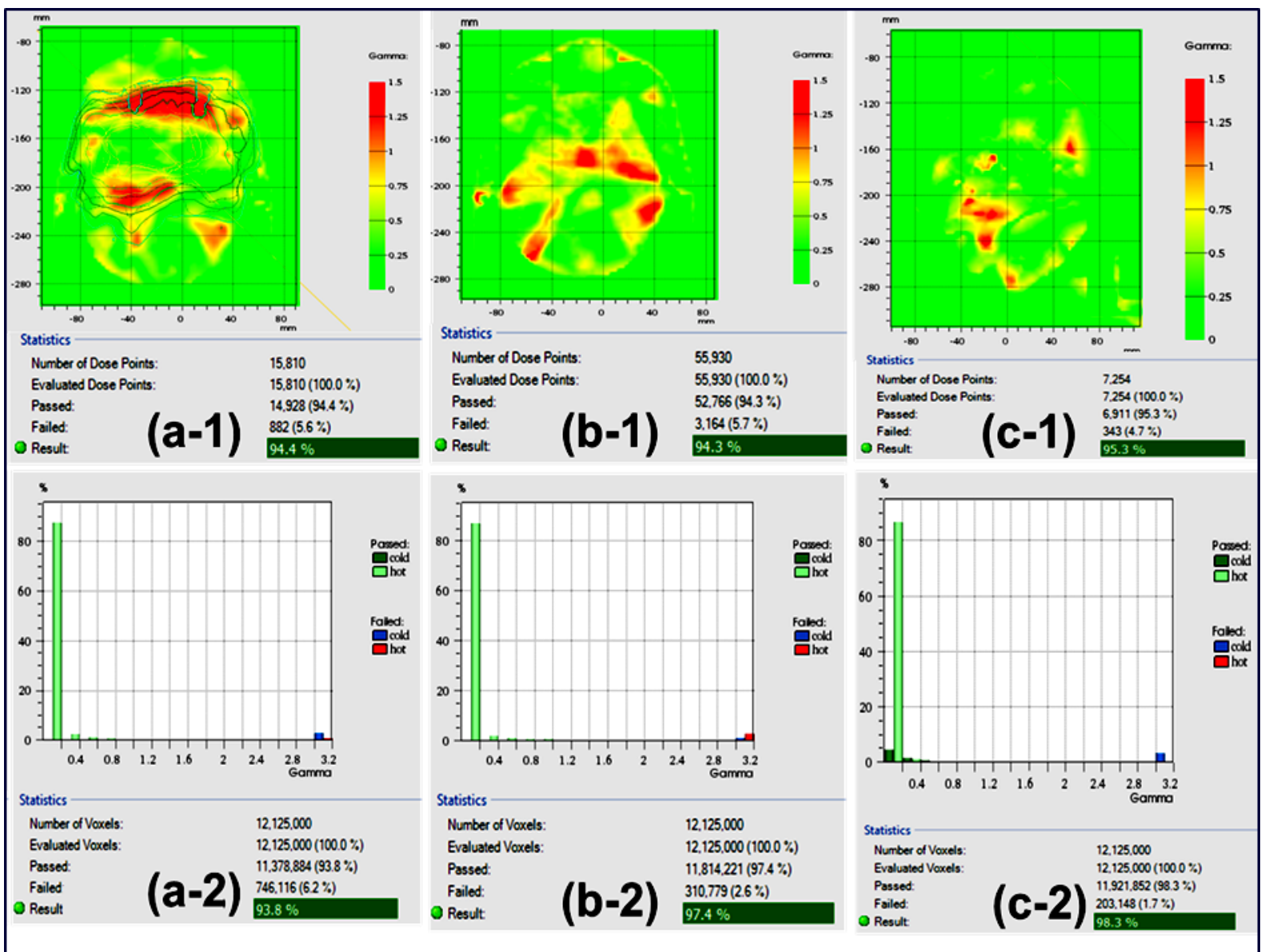

Figure 4. Comparison of 2D (a-1, b-1, c-1) and 3D (a-2, b-2, c-2) dose distribution between a) TPS and MC simulation with SOURCE 8 , b) TPS and simulation with SOURCE 20, and c) simulation with SOURCE 8 and SOURCE 20 in the DOSXYZnrc.

Again, for the 2D dose distribution, the Gamma index $(3 \% / 3 \mathrm{~mm})$ between SOURCE 8 and 20 (as shown in figure $4 \mathrm{c}-1$ ) is $95.3 \%$ with 4.7 difference. While for 3D dose distribution, Gamma index was $98.3 \%$ with $1.7 \%$ difference. A detailed comparison of Gamma index shown in table 1 and shows that SOURCE 20 has better conformity as compared to SOURCE 8.

Table 1. Summary of 3D gamma passing rate with different acceptance criteria.

\begin{tabular}{|c|c|c|c|}
\hline Criteria & $\begin{array}{c}\text { TPS VS } \\
\text { SOURCE } 8\end{array}$ & $\begin{array}{c}\text { TPS VS } \\
\text { SOURCE } 20\end{array}$ & $\begin{array}{l}\text { SOURCE } 8 \text { vS } \\
\text { SOURCE } 20\end{array}$ \\
\hline $3 \% / 3 \mathrm{~mm}$ & $93.8 \%$ & $94.7 \%$ & $98.3 \%$ \\
\hline $2 \% / 2 \mathrm{~mm}$ & $92.4 \%$ & $93.7 \%$ & $97.9 \%$ \\
\hline $1 \% / 1 \mathrm{~mm}$ & $91.2 \%$ & $93.0 \%$ & $97.5 \%$ \\
\hline
\end{tabular}

DVH

Comparison between TPS and MC simulations for dose values to PTV and organ-at- risk (OAR) shown is figure 5.

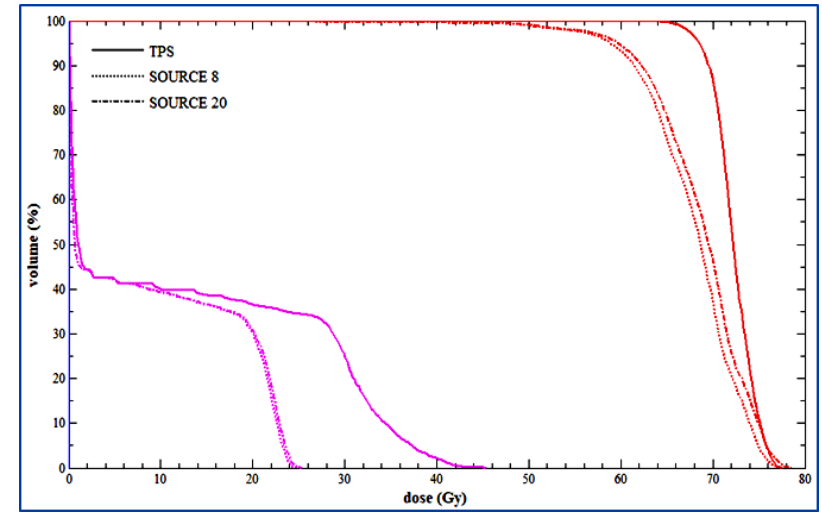

Figure 5. Dose volume histograms between TPS and MC simulation.

The difference between TPS and MC simulations may be due to the differences in algorithms used in dose calculation. While SOURCE 20 tends to be better than SOURCE 8 for 
both PTV and OAR. A detailed comparison shown in table 2.

Table 2. The dose value of PTV and the dose value to the spinal cord for TPS and MC simulation.

\begin{tabular}{|c|c|c|c|c|c|c|}
\hline \multirow{2}{*}{ Parameter } & \multicolumn{3}{|c|}{ PTV (Gy) } & \multicolumn{3}{c|}{ Spinal cord (Gy) } \\
\cline { 2 - 7 } & TPS & $\begin{array}{c}\text { SOURCE } \\
\mathbf{8}\end{array}$ & $\begin{array}{c}\text { SOURCE } \\
\mathbf{2 0}\end{array}$ & TPS & $\begin{array}{c}\text { SOURCE SOURCE } \\
\mathbf{8}\end{array}$ & $\mathbf{2 0}$ \\
\hline $\mathbf{D}_{\text {mean }}$ & 72.34 & 68.72 & 67.85 & 12.86 & 8.79 & 8.68 \\
\hline $\mathbf{D}_{\max }$ & 77.55 & 78.52 & 77.50 & 45.30 & 25.32 & 24.90 \\
\hline $\mathbf{D}_{\min }$ & 16.91 & 16.70 & 16.51 & 0.15 & 0.10 & 0.10 \\
\hline
\end{tabular}

\section{Time running and working load}

Two cases of simulated MC to change the structure of the SOURCE (SOURCE 8 and SOURCE 20 in the library SOURCE of the DOSXYZnrc) conducted on the same computer, the input parameters of interaction and transport of the beam identical. However, the workload required in the declaration and the process of simulation time for two SOURCEs is different; these comparison results presented in table 3 .

Table 3. Time running and working load between two different SOURCEs.

\begin{tabular}{|c|c|c|c|c|c|}
\hline \multirow{3}{*}{ Type } & \multicolumn{2}{|c|}{ BEAMnrc } & \multicolumn{2}{c|}{ DOSXYZnrc } & Total \\
\cline { 2 - 5 } & Work load & $\begin{array}{c}\text { Time } \\
\text { (h) }\end{array}$ & Work load & $\begin{array}{c}\text { Time } \\
\text { (h) }\end{array}$ & $\begin{array}{c}\text { (h) } \\
\text { (h) }\end{array}$ \\
\hline \multirow{3}{*}{$\begin{array}{c}\text { SOURCE } \\
8\end{array}$} & $\begin{array}{c}\text { Input 49 } \\
\text { BEAM for 49 } \\
\text { segments }\end{array}$ & 3.0 & $\begin{array}{c}\text { Input 49 } \\
\text { DosXYZnrc for } \\
49 \text { segments }\end{array}$ & 1.5 & \multirow{2}{*}{358.5} \\
\cline { 2 - 6 } & $\begin{array}{c}\text { Running 49 } \\
\text { times }\end{array}$ & 159.0 & $\begin{array}{c}\text { Running 49 } \\
\text { times }\end{array}$ & 195.0 & \\
\hline \multirow{2}{*}{$\begin{array}{c}\text { SOURCE } \\
20\end{array}$} & $\begin{array}{c}\text { Input 49 } \\
\text { segments in } \\
\text { BEAM }\end{array}$ & 0.5 & $\begin{array}{c}\text { Input 49 } \\
\text { segments in 1 } \\
\text { DosXYZnrc }\end{array}$ & 0.5 & \multirow{2}{*}{96.5} \\
\cline { 2 - 5 } & $\begin{array}{c}\text { Running } \\
\text { only 1 time }\end{array}$ & 55 & $\begin{array}{c}\text { Running only 1 } \\
\text { time }\end{array}$ & 40.5 & \\
\hline
\end{tabular}

For the first case with SOURCE 8, the declarations in the BEAMnrc and DOSXYZnrc must be done separately for each segment of each beam. Total runing time was 358.5 hours (about 15 days). That is not only time consuming and tedious but also error prone during the simulation process.

\section{DISCUSSION}

There are many SOURCE options available in the DOSXYZnrc to calculating the dose distribution by MC simulation. In our previous study (18), the JO-IMRT dose distributions calculated by using SOURCE 8 . Our results show that the average gamma passing rates were 93.3 $\pm 3.1 \%, 92.8 \pm 3.2 \%, 92.4 \pm 3.4 \%$ based on the $3 \% / 3 \mathrm{~mm}, 2 \% / 2 \mathrm{~mm}, 1 \% / 1 \mathrm{~mm}$ criteria and the simulation time for one case was about 16 days because 49 segments in J0-IMRT plan were simulated separately. An aspirational objective of this work was to compare SOURCE 8 and 20 in the DOSXYZnrc to find out the best SOURCE for calculating JO-IMRT by MC simulation. The isodose distributions on CT slices (figure 3), $\mathrm{DVH}$ of the two dose distributions (figure 5) calculated with the DOXYZnrc and obtained from the TPS used at Dong Nai General Hospital were compared using CERR (25). Gamma index $(1 \% / 1 \mathrm{~mm}, 2 \% / 2 \mathrm{~mm}$, and $3 \% / 3 \mathrm{~mm}$ ) in table 1 calculated by using PTW-Verisoft (figure 4). Furthermore, the efficient computation of dose distributions such as time running, working load and uncertainty error calculation were also evaluated (table 3).

When performing the gamma evaluation, it is generally expected that at least $90 \%$ gamma passing rate is within the tolerance. In this work, we found good agreement between MC and TPS with passing rates higher than $90 \%$ for $1 \% / 1$ $\mathrm{mm}, 2 \% / 2 \mathrm{~mm}$, and 3\%/ $3 \mathrm{~mm}$ criteria. Asuni et. al. (26) used SOURCE 20 in DOXYZnrc to calculating the dynamic IMRT dose distribution for head and neck patient that found the same our result.

Based on the dose distribution on each slice of the plans in figure 3, our observations that visually the co-dose on the same slice had a good fit. Two dose distributions from MC simulation gave relatively similar dose images and there was a fit between the two MC simulation cases.

According to table 2, the $\mathrm{D}_{\text {mean }}$ value of PTV 70 to TPS was 72.34 Gy while for MC simulations (for SOURCE 8 and 20) was 68.72 Gy and 
67.85Gy, respectively (local difference SOURCE 8 vs. TPS and SOURCE 20 vs. TPS were $5 \%$ and $0.63 \%$, respectively). There was no significant difference in these two types of SOURCEs in the JO-IMRT treatment.

The $D_{\max }$ to the spinal cord in the TPS was 12.86 Gy while the $D_{\max }$ to the spinal cord of MC simulations (for SOURCE 8 and 20) was $25.32 \mathrm{~Gy}$ and $24.90 \mathrm{~Gy}$, respectively. This number is much smaller than TPS and the difference between the two MC simulations is extremely small at only $1.63 \%$. Other work performed by Yani et al. (13, 16) found similar DVH results between MC and TPS. Our findings in this study demonstrated a very good potential for using SOURCE 20 in simulations especially JO-IMRT as well as other techniques.

From the comparison results in table 3 , the simulation time with SOURCE 8 is 3.7 times longer than that of SOURCE 20. The simulation for each segment with SOURCE 8 just only took more than 7 hours, but we had to perform the simulation for 48 segments remaining and process the data after the simulation, therefore, the total time required is very large. However, the most important and meaningful is not only minimizing the simulation time when using SOURCE 20 for simulation, but also allows the user to simultaneously declare all the input of the field of beams on the interface of the DOSXYZnrc, which simplify the declaration process and facilitate the modification. A similar comparison between SOURCE 20 and 21 showed that SOURCE 20 requires approximately 8 times less time with $0.2 \%$ uncertainty in high dose region (17).

\section{CONCLUSION}

In this study, a MC based JO-IMRT dose calculations performed with SOURCE 8 and 20 using theDOSXYZnrc. Isodose distributions on CT slices, DVH and Gamma Index compared with DOSXYZnrc and TPS. Results showed that SOURCE 20 is more efficient, accurate, and requires less time as compare to SOURCE 8 for IMRT dose distribution and can be used further for IMRT or VMAT dose distributions.

Int. J. Radiat. Res., Vol. 19 No. 4, October 2021

\section{ACKNOWLEDGMENT}

The authors would like to thank Professor James C.L. Chow, University of Toronto, Ontario, Canada for providing the DOSCTP and proofreading this manuscript. The authors also would like to extend thanks to associate professor Truong Thi Hong Loan and Dr. Nguyen Dong Son, VNUHCM-University of Science for the supervision of our MC Group.

Conflicts of interest: Declared none.

\section{REFERENCES}

1. Naqvi SA, Earl MA, Shepard DM (2003) Convolution/ superposition using the Monte Carlo method. Phys Med Biol, 48(14): 2101.

2. Krieger T, Sauer OA (2005) Monte Carlo-versus pencilbeam-/collapsed-cone-dose calculation in a heterogeneous multi-layer phantom. Phys Med Biol, 50(5): 859.

3. Kim SJ, Kim SK, Kim DH (2015) Comparison of pencil-beam, collapsed-cone and Monte-Carlo algorithms in radiotherapy treatment planning for 6-MV photons. J Korean Phy Soc, 67(1): 153-158.

4. Hasenbalg F, Neuenschwander H, Mini R, Born EJ (2007) Collapsed cone and analytical anisotropic algorithm dose calculations compared to VMC++ Monte Carlo simulations in clinical cases. In J Phys: Conf Ser, 21007.

5. Rogers DWO (2006) Fifty years of Monte Carlo simulations for medical physics. Phys Med Biol, 51(13): R287.

6. Seco J and Verhaegen F (2013) Monte Carlo techniques in radiation therapy. CRC press.

7. Reynaert N, Marck S, Schaart D (2006) Monte Carlo treatment planning-An introduction, Nederlandse Commissie Voor Stralingsdosimetrie.

8. Kawrakow I (2001) The EGSnrc code system, Monte Carlo simulati on of electron and photon transport. NRCC Report PIRS-701, Ottawa, Canad.

9. Hoang TD, Duong TT, Luong OT, et al. (2019) Application of variance reduction techniques in EGSnrc based MonteCarlo method. Science and Technology Development Journal, 22(2): 258-263.

10. Francescon P, Cora S, Chiovati P (2003) Dose verification of an IMRT treatment planning system with the BEAM EGS4-based Monte Carlo code. Med phys, 30(2): 144-157.

11. Benhalouche S, Visvikis D, Le Maitre A, et al. (2013) Evaluation of clinical IMRT treatment planning using the GATE Monte Carlo simulation platform for absolute and relative dose calculations. Med phys, 40(2): 021711.

12. Bergman AM, Gete E, Duzenli C, et al. (2014) Monte Carlo modeling of HD120 multileaf collimator on Varian True- 


\section{Tai et al. / A comparison of the source 8 and 20 in the DOSXYZnrc}

Beam linear accelerator for verification of $6 \mathrm{X}$ and $6 \mathrm{X}$ FFF VMAT SABR treatment plans. J Appl Clin Med Phys, 15(3): 148-163.

13. Yani S, Rizkia I, Rhani MF, Mohammad H, Freddy H (2020) EGSnrc application for IMRT planning. Rep Pract Oncol Radiother, 25(2): 217-226.

14. Rogers DWO, Walters B, Kawrakow I (2009) BEAMnrc users manual. NRC Report PIRS, 509, 12.

15. Walters BRBI, Kawrakow I, Rogers DWO (2005) DOSXYZnrc users manual. NRC Report PIRS, 794: 1-125.

16. Yani S, Rizkia I, Rhani MF, Mohammad H, Freddy H (2020) Study of efficiency in five-field and field-by-field intensity modulated radiation therapy (IMRT) plan using DOSXYZnrc Monte Carlo code. Rep Pract Oncol Radiother, 25(3): 428435.

17. Linares Rosales HM, Lara Mas E, Alfonso Laguardia R, Tony $P$ (2015) Application of a Monte Carlo linac model in routine verifications of dose calculations. Nucleus, 57.

18. Tai DT, Oanh LT, Son ND, Loan TTH, James CLC (2019) Dosimetric and Monte Carlo verification of jaws-only IMRT plans calculated by the Collapsed Cone Convolution algorithm for head and neck cancers. Rep Pract Oncol Radiother, 24(1): 105-114.

19. Oanh LT, Tai DT, Loan TTH, Minh TV, James CLC (2019) Dosimetric evaluation of lung treatment plans produced by the Prowess Panther system using Monte Carlo simulation. Biomed Phys Eng Express, 5(5): 055005.
20. Tai DT, Son ND, Loan TTH, Tuan HD (2017) A method for determination of parameters of the initial electron beam hitting the target in linac. J Phys: Conf Ser, 851(1): 012032.

21. Luong OT, Dang LT, Duong TT (2018) Method for calculation the absolute dose in the Monte Carlo simulation. Science and Technology Development Journal-Natural Sciences, 2(5): 90-96.

22. Lobo J, Popescu IA (2010) Two new DOSXYZnrc sources for 4D Monte Carlo simulations of continuously variable beam configurations, with applications to RapidArc, VMAT, TomoTherapy and CyberKnife. Phys Med Biol, 55(16): 4431.

23. Zhan L, Jiang R, Osei EK (2012) Beam coordinate transformations from DICOM to DOSXYZnrc. Phys Med Biol, $\mathbf{5 7}$ (24): N513.

24. Ebert MA and Spry NA (2001) Dose perturbation by air cavities in megavoltage photon beams: implications for cavity surface doses. Australas Radiol, 45(2): 205-210.

25. Deasy JO, Blanco Al, Clark VH (2003) CERR: a computational environment for radiotherapy research. Med Phys, 30 (5): 979-985.

26. Asuni G, Van Beek T, Venkataraman S, Popescu I A, McCurdy B M (2013) A Monte Carlo tool for evaluating VMAT and DIMRT treatment deliveries including planar detectors. Physics in Medicine \& Biology, 58(11): 3535. 